\title{
WEIGHTED TANGENTIAL BOUNDARY LIMITS OF SUBHARMONIC FUNCTIONS ON DOMAINS IN $\mathrm{R}^{n}(n \geq 2)$
}

\author{
MANFRED STOLL
}

\section{Abstract}

In the paper we consider weighted non-tangential and tangential boundary limits of non-negative subharmonic functions on bounded domains in $\mathrm{R}^{n}, n \geq 2$.

The main result of the paper is as follows: Let $f$ be a non-negative subharmonic function on a bounded domain $\Omega$ with $C^{1}$ boundary satisfying

$$
\int_{\Omega} \delta(y)^{\gamma} f^{p}(y) d y<\infty
$$

for some $p>0$, and some $\gamma>-1-\beta(p)$, where $\beta(p)=\max \{(n-1)(1-p), 0\}$ and $\delta(y)$ denotes the distance from $y$ to $\partial \Omega$. Suppose $\tau \geq 1$. Then for a.e. $\zeta \in \partial \Omega$,

$$
f^{p}(y)=o\left(\delta(y)^{\left(\frac{n-1}{\tau}\right)-\gamma-n}\right)
$$

uniformly as $y \rightarrow \zeta$ in each $\Gamma_{\tau, \alpha}(\zeta)$, where for $\alpha>0(\alpha>1$ when $\tau=1)$

$$
\Gamma_{\tau, \alpha}(\zeta)=\left\{y \in \Omega:|y-\zeta|^{\tau}<\alpha \delta(y)\right\} .
$$

\section{Introduction.}

The results of this paper were motivated by the following result of $\mathrm{F}$. W. Gehring [4] (see also [13, Theorem IV. 41]):

Theorem. Suppose $w(z)$ is a non-negative subharmonic function in the unit disc $|z|<1$ in C satisfying

$$
\iint_{|z|<1} w^{p}(z) d x d y<\infty, \quad z=x+i y,
$$

for some $p>1$. Then for almost every $\theta$, 


$$
w(z)=o\left((1-|z|)^{-1 / p}\right)
$$

uniformly as $z \rightarrow e^{i \theta}$ in each non-tangential approach region $\Gamma_{\alpha}\left(e^{i \theta}\right)$.

This last statement is equivalent to

$$
\lim _{r \rightarrow 1^{-}} \sup _{\substack{z \in \Gamma_{\alpha}\left(e^{i \theta}\right) \\|z| \geq r}}(1-|z|) w^{p}(z)=0
$$

for almost every $\theta$, where for $\alpha>1$,

$$
\Gamma_{\alpha}\left(e^{i \theta}\right)=\left\{z:\left|e^{i \theta}-z\right|<\alpha(1-|z|),|z|<1\right\} .
$$

The proof of the theorem used the Hardy-Littlewood theorem which accounts for the assumption that $p>1$.

Using techniques of potential theory we extend the previous theorem in several directions. First, we remove the restriction on $p>1$ and prove that the result of Gehring is valid for all $p, 0<p<\infty$. Second, we extend the result to subharmonic functions on bounded domains in $\mathrm{R}^{n}, n \geq 2$, with $C^{1}$ boundary. Finally, in addition to non-tangential limits, we will also consider weighted boundary limits along tangential approach regions.

For a bounded domain $\Omega \subset \mathrm{R}^{n}, n \geq 2$, and $x \in \Omega$, let $\delta(x)$ denote the distance from $x$ to $\partial \Omega$, the boundary of $\Omega$. The boundary of $\Omega$ is said to be $C^{1}$ if there exists a $C^{1}$ function $\rho: \mathbf{R}^{n} \rightarrow \mathrm{R}$ such that $\Omega=\left\{x \in \mathbf{R}^{n}\right.$ : $\rho(x)<0\}, \partial \Omega=\left\{x \in \mathbf{R}^{n}: \rho(x)=0\right\}$, and $\nabla \rho(x) \neq 0$ for all $x \in \partial \Omega$. This last condition ensures that at each $\zeta \in \partial \Omega$ there is a tangent plane and an outward unit normal, denoted by $\mathbf{n}_{\zeta}$.

Let $\zeta \in \partial \Omega$. For $\tau \geq 1$ and $\alpha>0(\alpha>1$ when $\tau=1)$, set

$$
\Gamma_{\tau, \alpha}(\zeta)=\left\{y \in \Omega:|y-\zeta|^{\tau}<\alpha \delta(y)\right\} .
$$

In the unit disc, when $\tau=1$ and $\alpha>1$, these are the non-tangential regions $\Gamma_{\alpha}$ defined above. As we will see below, when $\tau>1$, the regions $\Gamma_{\tau, \alpha}(\zeta)$ have tangential contact in all directions at $\zeta$.

Finally, as in [12], for $p>0$, set $\beta(p)=\max \{(n-1)(1-p), 0\}$. The main result of the paper is as follows:

THEOREM 1. Let $f$ be a non-negative subharmonic function on a bounded domain $\Omega$ with $C^{1}$ boundary satisfying

$$
\int_{\Omega} \delta(y)^{\gamma} f^{p}(y) d y<\infty
$$

for some $p>0$, and $\gamma>-1-\beta(p)$. Then for each $\tau \geq 1$ and $\alpha>0(\alpha>1$ when $\tau=1$ ) 


$$
\lim _{\rho \rightarrow 0} \sup _{y \in \Gamma_{\tau, \alpha, \rho}(\zeta)} \delta(y)^{n+\gamma-\left(\frac{n-1}{\tau}\right)} f^{p}(y)=0 \quad \text { for a.e. } \zeta \in \partial \Omega
$$

where $\Gamma_{\tau, \alpha, \rho}(\zeta)=\left\{y \in \Gamma_{\tau, \alpha}(\zeta): \delta(y)<\rho\right\}$.

The special case $n=2, \gamma=0$, and $\tau=1$ gives the result of Gehring in the setting of the unit disc. In the hypothesis of Theorem 1 we require that $\gamma>-1-\beta(p)$, since by Theorem 2 of [12], if $\gamma \leq-1-\beta(p)$, then the only non-negative subharmonic function $f$ satisfying (1.4) on a bounded domain with $C^{2}$ boundary for some $p>0$ is the zero function. The proof of Theorem 1 will be given in Section 3. In Section 4 we give two extensions of Theorem 1. The first is a restatement of Theorem 1 in terms of $d$-dimensional Hausdorff measure, while the second provides an extension to include unbounded domains. The analogue of Theorem 1 for functions that are subharmonic with respect to the Laplace-Beltrami operator on the unit ball in $C^{n}$ was proved by the author in [11].

Tangential boundary limits of harmonic functions or Green potentials have been considered by many authors, including Y. Mizuta [7, 8], A. Nagel, W. Rudin, and J. H. Shapiro [9], and J-M. G. Wu [14], among many others. A good reference for the numerous results concerning non-tangential and tangential boundary limits of Green potentials on the upper half-space in $\mathrm{R}^{n}$ is the paper by R. D. Berman and W. S. Cohn [1]. Many of the results involving tangential boundary limits of Green potentials were motivated by the results of G. T. Cargo [2] and J. R. Kinney [6] concerning tangential boundary limits of Blaschke products in the unit disc.

Many of the above referenced results involve tangential boundary limits in the half-space $\mathscr{H}$ in $\mathrm{R}^{n}$, where for $n \geq 2$,

$$
\mathscr{H}=\left\{\left(x^{\prime}, x_{n}\right): x^{\prime} \in \mathrm{R}^{n-1}, x_{n}>0\right\} .
$$

For $\zeta=\left(\zeta^{\prime}, 0\right) \in \partial \mathscr{H}, \tau \geq 1$, and $\beta>0(\beta>1$ when $\tau=1)$, set

$$
\mathscr{A}_{\tau, \beta}(\zeta)=\left\{y \in \mathscr{H}:|y-\zeta|^{\tau}<\beta y_{n}\right\} .
$$

When $\tau=1$ and $\beta>1, \mathscr{A}_{1, \beta}(\zeta)$ is an open cone at $\zeta$ with axis in the direction $\left(0^{\prime}, 1\right)$ and angle $\arccos \frac{1}{\beta}$. On the other hand, when $\tau=2, \beta>0$,

$$
\mathscr{A}_{2, \beta}(\zeta)=\left\{y \in \mathscr{H}:\left|y^{\prime}-\zeta^{\prime}\right|^{2}+\left(y_{n}-\frac{1}{2} \beta\right)^{2}<\left(\frac{1}{2} \beta\right)^{2}\right\}=B_{\frac{1}{2} \beta}\left(\zeta^{\prime}, \frac{1}{2} \beta\right),
$$

where $B_{r}(x)$ is the open ball of radius $r$ centered at $x$.

As we will see, the approach regions $\Gamma_{\tau, \alpha}(\zeta)$ are very similar to the regions $\mathscr{A}_{\tau, \alpha}$. Fix $\zeta \in \partial \Omega$. By translation and rotation we can assume without loss of generality that $\zeta=0$, and that in a neighborhood $U$ of $0, \Omega$ is given by

$$
U \cap \Omega=\left\{\left(y^{\prime}, y_{n}\right) \in U: y^{\prime} \in V, y_{n}>\varphi\left(y^{\prime}\right)\right\},
$$


where $V$ is a neighborhood of $0^{\prime}$ in $\mathrm{R}^{n-1}, \varphi$ is a $C^{1}$ function defined on $V$ with $\varphi\left(0^{\prime}\right)=0$ and $\nabla \varphi\left(0^{\prime}\right)=0^{\prime}$. For purposes of illustration we assume that $\varphi\left(y^{\prime}\right) \geq 0$ for all $y^{\prime} \in V$. If $\varphi\left(y^{\prime}\right)=0$ for all $y^{\prime} \in V$, then $\Gamma_{\tau, \alpha}(0)=\mathscr{A}_{\tau, \alpha}(0)$. Since $\varphi$ is assumed to be non-negative, we have $\delta(y) \leq y_{n}$ for all $y \in U \cap \Omega$. Thus

$$
\Gamma_{\tau, \alpha}(0) \cap U \subset \mathscr{A}_{\tau, \alpha}(0) \cap U
$$

for all $\tau \geq 1$ and $\alpha>0(\alpha>1$ when $\tau=1)$. If $\varphi\left(y^{\prime}\right) \leq 0$, then the reverse containment holds.

Since $\partial \Omega$ is $C^{1}$, there exists $\beta_{o}>1$ such that $\overline{\mathscr{A}}_{1, \beta}(0) \cap \partial \Omega \cap U=\{0\}$ for all $\beta, 1<\beta \leq \beta_{o}$. If in addition, $\partial \Omega$ is $C^{1, \gamma}(0<\gamma \leq 1)$ near 0 , that is, there exists a positive constant $C$ such that

$$
\left|\nabla \varphi\left(\zeta^{\prime}\right)-\nabla \varphi\left(\xi^{\prime}\right)\right| \leq C\left|\zeta^{\prime}-\xi^{\prime}\right|^{\gamma}
$$

for all $\zeta^{\prime}, \xi^{\prime} \in V$, then there exists $\beta_{o}>0$ such that

$$
\overline{\mathscr{A}}_{1+\gamma, \beta}(0) \cap \partial \Omega \cap U=\{0\}
$$

for all $\beta, 0<\beta \leq \beta_{0}$. This is an immediate consequence of the fact that if $\varphi$ is $C^{1, \gamma}$, then $\left|\varphi\left(\zeta^{\prime}\right)\right| \leq C\left|\zeta^{\prime}\right|^{1+\gamma}$ for all $\zeta^{\prime} \in V$.

Suppose now that $\tau \geq 1$ and $\beta_{o}>0\left(\beta_{o}>1\right.$ if $\left.\tau=1\right)$ is such that

$$
\overline{\mathscr{A}}_{\tau, \beta_{o}}(0) \cap \partial \Omega \cap U=\{0\} .
$$

We will show that if this is the case, then given $\alpha>0$, there exists $\beta_{\alpha}<\beta_{o}$ such that

$$
\mathscr{A}_{\tau, \beta}(0) \cap U \subset \Gamma_{\tau, \alpha}(0) \cap U
$$

for all $\beta \leq \beta_{\alpha}$. Let $y \in \mathscr{A}_{\tau, \beta}(0), \beta<\beta_{o}$, and let $\zeta \in \partial \Omega \cap U$ be such that $\delta(y)=|\zeta-y|$. Then $y=\zeta-\delta(y) \mathbf{n}_{\zeta}$. Hence if we write $y=\left(y^{\prime}, y_{n}\right)$, we have $y_{n}=\varphi\left(\zeta^{\prime}\right)+\delta(y) / A$, where $A=\sqrt{\left|\nabla \varphi\left(\zeta^{\prime}\right)\right|^{2}+1}$. Since $\zeta \in \partial \Omega,|\zeta|^{\tau} \geq \beta_{o} \varphi\left(\zeta^{\prime}\right)$. Thus

$$
\begin{aligned}
|y|^{\tau}<\beta y_{n} & <\left(\frac{\beta}{\beta_{o}}\right)|\zeta|^{\tau}+\beta \delta(y) \leq\left(\frac{\beta}{\beta_{o}}\right)(|y|+\delta(y))^{\tau}+\beta \delta(y) \\
& <\left(\frac{\beta}{\beta_{o}}\right)\left(|y|^{\tau}+\tau 2^{\tau-1}|y|^{\tau-1} \delta(y)\right)+\beta \delta(y) \\
& <\left(\frac{\beta}{\beta_{o}}\right)|y|^{\tau}+\beta c \delta(y)
\end{aligned}
$$

for some positive constant $c$. Hence 


$$
|y|^{\tau}<\left(\frac{\beta \beta_{o} c}{\beta_{o}-\beta}\right) \delta(y) .
$$

From this it now follows that there exists $\beta_{\alpha}<\beta_{o}$ such that $\mathscr{A}_{\tau, \beta}(0) \subset \Gamma_{\tau, \alpha}(0)$ for all $\beta \leq \beta_{\alpha}$.

\section{Preliminaries.}

Prior to proving Theorem 1 we first state and prove several preliminary results. As in the Introduction, for $\zeta \in \partial \Omega, \tau \geq 1$, and $\alpha>0(\alpha>1$ if $\tau=1)$, set

$$
\Gamma_{\tau, \alpha}(\zeta)=\left\{y \in \Omega:|y-\zeta|^{\tau}<\alpha \delta(y)\right\} .
$$

Also, for $x \in \Omega$, let

$$
B(x)=B\left(x, \frac{1}{3} \delta(x)\right)=\left\{y \in \Omega:|x-y|<\frac{1}{3} \delta(x)\right\} .
$$

Lemma 1.

(a) For all $y \in B(x), \quad \frac{2}{3} \delta(x) \leq \delta(y) \leq \frac{4}{3} \delta(x)$.

(b) Let $\zeta \in \partial \Omega$. Suppose $\tau \geq 1, \alpha>0(\alpha>1$ when $\tau=1)$. Then there exists $\alpha^{\prime}>\alpha$ such that $B(x) \subset \Gamma_{\tau, \alpha^{\prime}}(\zeta)$ for all $x \in \Gamma_{\tau, \alpha}(\zeta)$ with $\delta(x) \leq 1$.

Proof. (a) Let $y \in B(x)$. Suppose $\zeta \in \partial \Omega$ is such that $|x-\zeta|=\delta(x)$. Then

$$
\delta(y) \leq|y-\zeta| \leq|y-x|+|x-\zeta|<\frac{4}{3} \delta(x) .
$$

On the other hand, if $\xi \in \partial \Omega$ is such that $|y-\xi|=\delta(y)$, then

$$
\delta(x) \leq|x-\xi| \leq|x-y|+|y-\xi|<\frac{1}{3} \delta(x)+\delta(y) .
$$

Thus $\delta(y) \geq \frac{2}{3} \delta(x)$.

(b) Suppose $x \in \Gamma_{\tau, \alpha}(\zeta)$ with $\delta(x) \leq 1$. Then for $y \in B(x)$,

$$
\begin{aligned}
|y-\zeta|^{\tau} & \leq(|y-x|+|x-\zeta|)^{\tau} \\
& \leq 2^{\tau}\left(|y-x|^{\tau}+|x-\zeta|^{\tau}\right) \\
& \leq\left(\frac{2}{3}\right)^{\tau} \delta(x)^{\tau}+2^{\tau} \alpha \delta(x),
\end{aligned}
$$

which by (a),

$$
\leq \alpha^{\prime} \delta(y)
$$

for some $\alpha^{\prime}>\alpha$.

For $y \in \Omega$, let

$$
\widetilde{\Gamma}_{\tau, \alpha}(y)=\left\{\zeta \in \partial \Omega: y \in \Gamma_{\tau, \alpha}(\zeta)\right\} .
$$

Also, for $\zeta \in \partial \Omega$ and $r>0$, let 


$$
S(\zeta, r)=\{\xi \in \partial \Omega:|\zeta-\xi|<r\} .
$$

If $\partial \Omega$ is $C^{1}$ and $\sigma$ denotes surface area measure on $\partial \Omega$, then there exists a positive constant $C$ such that $\sigma(S(\zeta, r)) \leq C r^{n-1}$ for all $\zeta \in \partial \Omega, r>0$.

Lemma 2. Let $\Omega$ be a bounded domain with $C^{1}$ boundary. If $y \in \Gamma_{\tau, \alpha}(\zeta)$, then

$$
\sigma\left(\widetilde{\Gamma}_{\tau, \alpha}(y)\right) \leq C \delta(y)^{(n-1) / \tau},
$$

where $C$ is a positive constant depending only on $\tau$ and $\alpha$.

Proof. Suppose $y \in \Gamma_{\tau, \alpha}(\zeta)$. If $\xi \in \widetilde{\Gamma}_{\tau, \alpha}(y)$, then

$$
|\xi-\zeta| \leq|\xi-y|+|y-\zeta| \leq 2 \alpha^{1 / \tau} \delta(y)^{1 / \tau} .
$$

Therefore $\widetilde{\Gamma}_{\tau, \alpha}(y) \subset S\left(\zeta, c \delta(y)^{1 / \tau}\right)$, with $c=2 \alpha^{1 / \tau}$. Thus

$$
\sigma\left(\widetilde{\Gamma}_{\tau, \alpha}(y)\right) \leq \sigma\left(S\left(\zeta, c \delta(y)^{1 / \tau}\right)\right) \leq C \delta(y)^{(n-1) / \tau} .
$$

The following generalization of an inequality of Fefferman and Stein will be crucial in the proof of Theorem 1.

LeMma 3. Let $\Omega$ be a proper open subset of $\mathrm{R}^{n}$, and let $f$ be a non-negative subharmonic function on $\Omega$. Then there exists a constant $C(n, p)$, depending only on $n$ and $p$, such that

$$
f^{p}(x) \leq \frac{C(n, p)}{\delta(x)^{n}} \int_{B(x)} f^{p}(y) d y
$$

for all $p>0$.

REMARK. Inequality (2.1) has previously been stated by Riihentaus in [10] and by Susuki in [12] For $p \geq 1$, the inequality follows immediately from the mean value property of subharmonic functions. For $0<p<1$, inequality (2.1) was proved in [3] for $|h|$, where $h$ is harmonic on $\Omega$. The same proof also works for non-negative subharmonic functions, and thus is omitted.

\section{Proof of Theorem 1.}

For $\rho>0$, set $\Omega_{\rho}=\{x \in \Omega: \delta(x)<\rho\}$, and

$$
\Gamma_{\tau, \alpha, \rho}(\zeta)=\Gamma_{\tau, \alpha}(\zeta) \cap \Omega_{\rho} .
$$

Also, for $\zeta \in \partial \Omega, \rho>0$, set

$$
M_{\rho}(\zeta)=\sup \left\{\delta(x)^{n+\gamma-\left(\frac{n-1}{\tau}\right)} f^{p}(x): x \in \Gamma_{\tau, \alpha, \rho}(\zeta)\right\} .
$$

By Lemmas 1 and 3, if $x \in \Gamma_{\tau, \alpha, \rho}(\zeta)$, 


$$
\begin{aligned}
\delta(x)^{n+\gamma-\left(\frac{n-1}{\tau}\right)} f^{p}(x) & \leq C \int_{B(x)} \delta(y)^{\gamma-\left(\frac{n-1}{\tau}\right)} f^{p}(y) d y \\
& \leq C \int_{\Gamma_{\tau, \alpha^{\prime}, \rho^{\prime}}(\zeta)} \delta(y)^{\gamma-\left(\frac{n-1}{\tau}\right)} f^{p}(y) d y,
\end{aligned}
$$

where $\alpha^{\prime}>\alpha$ and $\rho^{\prime}=\frac{4}{3} \rho$. Thus

$$
M_{\rho}(\zeta) \leq C \int_{\Omega_{\rho^{\prime}}} \chi_{\Gamma_{\tau, \alpha^{\prime}}(\zeta)}(y) \delta(y)^{\gamma-\left(\frac{n-1}{\tau}\right)} f^{p}(y) d y
$$

where for a set $E, \chi_{E}$ denotes the characteristic function of $E$. Integrating over $\partial \Omega$ with respect to surface area measure $\sigma$ gives

$$
\int_{\partial \Omega} M_{\rho}(\zeta) d \sigma(\zeta) \leq C \int_{\partial \Omega} \int_{\Omega_{\rho^{\prime}}} \chi_{\Gamma_{\tau, \alpha^{\prime}}(\zeta)}(y) \delta(y)^{\gamma-\left(\frac{n-1}{\tau}\right)} f^{p}(y) d y d \sigma(\zeta),
$$

which by Fubini's theorem and Lemma 2,

$$
\begin{aligned}
& \leq C \int_{\Omega_{\rho^{\prime}}} \sigma\left(\widetilde{\Gamma}_{\tau, \alpha^{\prime}}(y)\right) \delta(y)^{\gamma-\left(\frac{n-1}{\tau}\right)} f^{p}(y) d y \\
& \leq C \int_{\Omega_{\rho^{\prime}}} \delta(y)^{\gamma} f^{p}(y) d y
\end{aligned}
$$

If $f$ satisfies (1.4), then

$$
\lim _{\rho \rightarrow 0} \int_{\Omega_{\frac{4}{3} \rho}} \delta(y)^{\gamma} f^{p}(y) d y=0 .
$$

Thus if we let $M(\zeta)=\lim _{\rho \rightarrow 0} M_{\rho}(\zeta)$, by Fatou's lemma and the above,

$$
\int_{\partial \Omega} M(\zeta) d \sigma(\zeta) \leq \lim _{\rho \rightarrow 0} C \int_{\Omega_{\rho^{\prime}}} \delta(y)^{\gamma} f^{p}(y) d y=0 .
$$

Hence $M(\zeta)=0$ a.e. on $\partial \Omega$. Thus

$$
\lim _{\rho \rightarrow 0} \sup _{y \in \Gamma_{\tau, \alpha, \rho}(\zeta)} \delta(y)^{n+\gamma-\left(\frac{n-1}{\tau}\right)} f^{p}(y)=0
$$

for a.e. $\zeta \in \partial \Omega$.

\section{Extensions of Theorem 1.}

We conclude the paper by giving two extensions of Theorem 1 . Since the proofs follow easily from what has already been presented, they are omitted. 
Our first result is a restatement of Theorem 1 in terms of $d$-dimensional Hausdorff measure $H_{d}$.

THEOREM 2. Let $f$ be a non-negative subharmonic function on a bounded domain $\Omega$ with $C^{1}$ boundary satisfying

$$
\int_{\Omega} \delta(y)^{\gamma} f^{p}(y) d y<\infty
$$

for some $p>0$, and $\gamma>-1-\beta(p)$. Let $0<d \leq n-1$. Then for each $\tau \geq 1$, there exists a subset $E_{\tau}$ of $\partial \Omega$ with $H_{d}\left(E_{\tau}\right)=0$ such that

$$
\lim _{\rho \rightarrow 0} \sup _{y \in \Gamma_{\tau, \alpha, \rho}(\zeta)} \delta(y)^{n+\gamma-\left(\frac{d}{\tau}\right)} f^{p}(y)=0 \quad \text { for all } \zeta \in \partial \Omega \backslash E_{\tau} .
$$

The proof of Theorem 2 follows in the same way as Theorem 1, except that surface area measure is replaced by a measure $\nu$ on $\partial \Omega$ satisfying $\nu(S(\zeta, r)) \leq C r^{d}$ for all $\zeta \in \partial \Omega$.

Since the proof of Theorem 1 only involves the local boundary behavior of $f$, Theorem 1 is also valid for unbounded domains. Thus we have

THEOREM 3. Let $\Omega$ be a domain with $C^{1}$ boundary, and let $f$ be a non-negative subharmonic function on $\Omega$ satisfying

$$
\int_{\Omega \cap\{|y| \leq r\}} \delta(y)^{\gamma} f^{p}(y) d y<\infty
$$

for some $p>0, \gamma>-1-\beta(p)$, and every $r>0$. Then, for each $\tau \geq 1$ and $\alpha>0(\alpha>1$ when $\tau=1)$,

$$
\lim _{\rho \rightarrow 0} \sup _{y \in \Gamma_{\tau, \alpha, \rho}(\zeta)} \delta(y)^{n+\gamma-\left(\frac{n-1}{\tau}\right)} f^{p}(y)=0 \quad \text { for a.e. } \zeta \in \partial \Omega .
$$

\section{REFERENCES}

1. R. D. Berman and W. S. Cohn, Littlewood theorems for limits and growth of potentials along level sets of Hölder continuous functions, Amer. J. Math. 114 (1991), 185-227.

2. G. T. Cargo, Angular and tangential limits of Blaschke products and their successive derivatives, Canad. J. Math. 14 (1962), 334-348.

3. C. Fefferman and E. Stein, $H^{p}$ spaces ofseveral variables, Acta Math 129 (1972), 137-193.

4. F. W. Gehring, On the radial order of subharmonic functions, J. Math. Soc. Japan 9 (1957), 77-79.

5. G. H. Hardy and J. E. Littlewood, Some properties of conjugate functions, J. Reine Angew. Math. 167 (1932), 403-423. 
6. J. R. Kinney, Boundary behavior of Blaschke products in the unit circle, Proc. Amer. Math. Soc. 12 (1961), 484-488.

7. Y. Mizuta, On the boundary limits of harmonic functions with gradient in $L^{p}$, Ann. Inst. Fourier, Grenoble 34 (1984), 99-109.

8. Y. Mizuta, On the boundary limits of harmonic functions, Hiroshima Math. J. 18 (1988), $207-217$.

9. A. Nagel, W. Rudin, and J. H. Shapiro, Tangential boundary behavior of functions in Dirichlet-type spaces, Ann. of Math. 116 (1982), 331-360.

10. J. Riihentaus, On a theorem of Avanissian-Arsove, Exposition. Math. 7 (1989), 69-72.

11. M. Stoll, Boundary limits and non-integrability of $\mathscr{M}$-subharmonic functions on the unit ball in $\mathrm{C}^{n}$, Trans. Amer. Math. Soc. 349 (1997), 3773-3785.

12. N. Suzuki, Nonintegrability of harmonic functions in a domain, Japan J. Math. 16 (1990), 269-278.

13. M. Tsuji. Potential Theory in Modern Function Theory, Chelsea Publ. Co., New York, N.Y. 1975.

14. J.-M. G. Wu, $L^{p}$-densities and boundary behavior of Green potentials, Indiana Univ. Math. J. 28 (1979), 895-911.

\author{
DEPARTMENT OF MATHEMATICS \\ UNIVERSITY OF SOUTH CAROLINA \\ COLUMBIA, SC 29208 \\ USA \\ Email: stoll@math.sc.edu
}

